\title{
Les forêts et les montagnes vaudoises
}

Un retour sur la marginalité économique de l'incultum du Canton de Vaud entre Moyen Âge et époque moderne

Forest and mountain regions in Vaud: a contribution about the economic marginality of the incultum of Canton de Vaud between the Middle Ages and the modern period

\section{Alice Vanetti}

\section{(2) OpenEdition}

\section{Journals}

Édition électronique

URL : http://journals.openedition.org/artefact/3441

DOI : $10.4000 /$ artefact.3441

ISSN : 2606-9245

Éditeur :

Association Artefact. Techniques histoire et sciences humaines, Presses universitaires du Midi

Édition imprimée

Date de publication : 15 mars 2019

Pagination : 267-282

ISBN : 978-2-8107-0623-5

ISSN : 2273-0753

Référence électronique

Alice Vanetti, «Les forêts et les montagnes vaudoises 》, Artefact [En ligne], 9 | 2018, mis en ligne le 04 mars 2020, consulté le 28 novembre 2020. URL : http://journals.openedition.org/artefact/3441 ; DOI : https://doi.org/10.4000/artefact.3441

\section{(c)}

Artefact, Techniques, histoire et sciences humaines est mise à disposition selon les termes de la Licence Creative Commons Attribution - Pas d'Utilisation Commerciale - Pas de Modification 4.0 International. 


\section{Les forêts et les montagnes vaudoises}

Un retour sur la marginalité économique de l'incultum
du Canton de Vaud entre Moyen Âge et époque moderne Alice Vanetti

\section{Résumé}

L'incultum vaudois, soit ses régions non cultivées, a toujours été considéré comme marginal, lié à une économie pastorale et présentant des productions artisanales ponctuelles. La découverte de vestiges liés à une exploitation importante du territoire en vue de la production de produits comme fer, bois et surtout chaux, remet en question cette définition. À travers une approche pluridisciplinaire, nous nous proposons de rouvrir le débat sur le rôle de ces territoires marginaux au niveau du Canton et des communautés locales.

\section{Mots-clés}

archéologie de la production artisanale, exploitation forestière, économie des zones marginales, fours à chaux, incultum, Lidar

95 Alice Vanetti, « Les forêts et les montagnes vaudoises : un retour sur la marginalité économique de l'incultum du Canton de Vaud entre Moyen Âge et époque moderne », Artefact, 9, 2018, p. 267-282. 


\section{Forest and mountain regions in Vaud: a contribution about the economic marginality of the incultum of Canton de Vaud between the Middle Ages and the modern period}

\section{Abstract}

Vaud incultum-uncultivated land-has traditionally being considered marginal, characterized by a pastoral economy, and by some isolated artisanal productions. This assumption is called into question by the discovery of evidence of an important exploitation of the territory, aimed at the production of products such as iron, wood, and especially lime. By applying an interdisciplinary approach, we propose to open again the debate on the role of these marginal regions at the cantonal and local community levels.

\section{Keywords}

archaeology of craft production, lime kiln, lumbering, Lidar, economy of marginal regions, incultum 


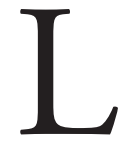
e canton de Vaud se situe dans la partie occidentale de la Suisse. Le plateau, au centre, est bordé par la chaîne du Jura au nord-ouest, les Alpes à l'est et les rives du Léman au sud. Canton le plus peuplé de la partie francophone de la Suisse, ou Suisse romande, il est le troisième de toute la Confédération. Il renferme plusieurs villes de tailles différentes, dont Lausanne, la capitale, la plus vaste d'entre elles. Il se caractérise par une forte diversité géographique due au fait qu'il touche, comme on l'a mentionné, trois aires. Malgré la présence de deux zones montagneuses, le canton de Vaud est plutôt un territoire de plateau que de montagne : le plateau occupe au moins les $63 \%$ de la surface vaudoise, contre $15 \%$ pour le Jura et $22 \%$ pour les Alpes. C'est pour ces raisons qu'il a été traditionnellement considéré comme un territoire à vocation essentiellement agricole, peu développé au niveau économique au moins jusqu'à la révolution industrielle. En 1972, par exemple, dans l'Encyclopédie illustrée du Pays de Vaud, l'économie vaudoise est à l'époque moderne, définie comme " dominée ", où " en dépit d'un artisanat actif, [l'on] exporte des produits agricoles (fromage, vin, blé, bétail) et [l’on] importe surtout des produits finis (tissus) ${ }^{1}$ ». Les forêts et les montagnes, présentes surtout dans la région du Jura et dans les zones qui composent les Alpes vaudoises, le Chablais et le Pays d'Enhaut, ont dans ce contexte souvent été considérées, par la recherche scientifique, comme des zones marginales. Les forêts servaient principalement à « fournir, par éradication progressive, une part importante des terres cultivables ${ }^{2}$ " et pour affourager le bétail. Les montagnes, bien que riches en matières minérales (calcaire, gravières, molasse, ainsi que minerai de fer), se caractérisaient par l'isolement et l'autoconsommation.

Entre les années 1950 et 1970, plusieurs historiens spécialistes d'histoire économique, tels que Robert Jaccard ${ }^{3}$ (1897-1980), Émile Buxcel ${ }^{4}$ (19282014), ou Paul-Louis Pelet (1920-2009) s'attachent à réévaluer le rôle de ces zones dans la vie économique régionale. Auteurs d'ouvrages centrés surtout sur le Jura vaudois, ces auteurs mettent en évidence grâce à l'exploitation en premier lieu de documents d'archives, la présence de plusieurs

1. Georges Nicolas-Obadia, "L'ancienne économie agraire ", in Henri Rieben (dir.), Les artisans de la prospérité, Encyclopédie illustrée vaudoise, n 3, 1972, p. 5.

2. Ibid., p. 16.

3. Robert JaCCARD, La Révolution industrielle dans le Canton de Vaud. Étude d'histoire économique, Lausanne, 1959.

4. Emile Buxcel, Aspects de la structure économique vaudoise 1803-1850, Lausanne, Bibliothèque historique vaudoise, 1981. 
petites unités de production dans toute la région. Ces unités auraient eu un rôle important, bien plus qu'on ne le croyait, dans l'industrialisation du canton réalisée à la fin du XIX ${ }^{\mathrm{e}}$ siècle. Buxcel, par exemple, met l'accent sur l'existence au milieu du XIx ${ }^{\mathrm{e}}$ siècle de plusieurs activités non-agricoles, dont une bonne partie qui utilise l'énergie des cours d'eau, déjà établies et florissantes avant ce qu'il appelle le " grand bond en avant " de l'industrialisation vaudoise. Paul-Louis Pelet, ensuite, professeur d'histoire économique à l'université de Lausanne, démontre, par le dépouillement d'archives antérieures à 1800, ainsi que par des campagnes de prospections et de fouilles, l'existence sur tout le canton d'une "multitude d'activités autres qu'agricoles ${ }^{5}$ ", dont la plupart sont précisément établies dans les mêmes zones non cultivées et considérées jusqu'alors comme quasiment non productives. Il étudie surtout la sidérurgie, qu'il définit davantage comme une activité rentable aux caractères proto-industriels que comme un artisanat rural permanent ${ }^{6}$. Les découvertes archéologiques réalisées par l'Archéologie cantonale au cours des dernières années renforcent ces conclusions, mais surtout l'enrichissent en mettant au jour la présence de plusieurs activités artisanales sur tout le canton ${ }^{7}$.

Situés principalement en forêt, loin des centres d'habitat et apparemment des principaux axes de communication, ces vestiges sont variés. En plus des lieux de production du fer, on observe de multiples sites liés aux cycles de production de la chaux et du verre, d'autres qui concernent l'extraction de la pierre à bâtir et le travail du bois. Pour ces derniers, les témoins matériels sont toutefois plus rares. Les restes découverts se retrouvent partout, en particulier sur le territoire du Jura. Ce qui frappe surtout le chercheur, c'est la quantité de structures : les sites qui ont pu être fouillés présentent plusieurs structures qui attestent la continuité de production pour une période prolongée. En ce qui concerne la production de la chaux, les fouilles ont confirmé la présence, sur le même site, de plusieurs fours pour la cuisson

\footnotetext{
5. Paul Louis Pelet, «Une industrie bimillénaire : la sidérurgie du Jura vaudois », Annales. Histoire, Sciences Sociales, 1974, p. 789-812.

6. Ibid.

7. Nous préférons employer le terme d'artisanat en tant que production vouée à la transformation d'un produit grâce à un savoir-faire particulier et non pas celui de proto-industrie puisque les données aujourd'hui à notre disposition ne nous permettent pas d'attribuer ce concept aux sites de productions relevés sur les territoires marginaux vaudois. À partir des recherches exposées dans cet article, un possible scénario proto-industriel se dessine, mais cela demande la mise en place de recherches ultérieures et plus approfondies.
} 
du calcaire, dans la plupart des cas en fonction entre le $\mathrm{XV}^{\mathrm{e}}$ et le XVIII ${ }^{\mathrm{e}}$ siècle d'après la datation réalisée au carbone 14 (Fig. VIII, cahier couleur). Cela démentirait en partie l'interprétation d'une production ponctuelle souvent attribuée à ce genre d'activités. Ensuite, la découverte, tant dans la région du Jura que dans le Chablais, de sites appartenant à des phases différentes de la production (des carrières pour l'extraction de la pierre ou des mines pour celle du minerai de fer, jusqu'aux fours pour la cuisson, aux moulins pour l'alimentation de scieries ou aux restes des forges) démontrent l'importance de l'exploitation de ces territoires, qui ne s'arrêterait pas à l'exploitation des matières premières mais couvrirait toutes les phases des chaînes de production. On peut donc se poser la question du rôle effectif de ces zones, incultivées et supposément marginales, au niveau de l'économie du pays de Vaud.

Tant le Jura que le Chablais et le pays d'Enhaut sont des territoires dont l'économie a surtout été de type pastoral. Dotés d'un vaste domaine forestier situé à des altitudes élevées, ils ont été défrichés au fur et à mesure pour en faire des pâturages destinés aux ovins, aux caprins et aux suidés. La population représente un faible pourcentage de celle du canton : les données d'aujourd'hui (et les sources historiques et archéologiques ne contredisent pas ces résultats) représentent $6,6 \%$ pour les Alpes et 4,3\% pour le Jura. $\mathrm{Au}$ niveau géologique, ces régions constituent néanmoins une importante source de matériaux de construction (les calcaires sont les constituants principaux de la chaîne du Jura et des Préalpes) et de minerais, surtout de fer, bien que la Suisse reste un pays " pauvre de gisements en minerais rentables $^{8}$ ». De plus, la grande densité en forêts, au-delà d'offrir un terrain pour la chasse et pour le pâturage, procure une grande disponibilité de bois, l'une des matières premières les plus employées pour la construction et pour la production du charbon, par exemple. Ces territoires ont donc un environnement favorable au développement d'activités artisanales basées sur l'exploitation de ces multiples matières premières. Pourtant, lorsqu'on essaie de déchiffrer l'ampleur du phénomène artisanal et son importance pour les sociétés et pour l'économie locale et générale du canton, on peine à affirmer sa primauté par rapport à d'autres formes de subsistance comme le pastoralisme. Comme nous l'avons affirmé, les évidences archéologiques qui attestent de l'exploitation des matières premières en vue d'une

8. Werner Belllwald, "Ressources minières ", dans Dictionnaire historique de la Suisse (DHS), 2010, en ligne : http://www.hls-dhs-dss.ch/textes/f/F13917.php, consulté le 13 novembre 2018. 
production artisanale sont nombreuses. Seules certaines d'entre elles sont néanmoins fixées sur un lieu précis et sont continues sur plusieurs années. C'est le cas de l'artisanat du fer. Déjà Pelet avait indiqué son importance primordiale pour ces régions. Son rôle a effectivement été confirmé par les découvertes archéologiques récentes. Dans le cas des puits de l'Auberson, sur la commune de Sainte-Croix, l'exploration d'un puits de mine, coffré de madriers d'épicéas liés à mi-bois aux angles, a non seulement révélé la présence d'une portion d'échelle encore en place, mais aussi d'une soixantaine d'éléments de bois travaillés qui avaient été rejetés dans ce puits ${ }^{9}$. Les datations dendrochronologiques ont mis en évidence la présence d'un véritable site d'extraction du fer en activité sur plusieurs années. Le décapage du pourtour du puits a révélé plusieurs zones spécifiques de travail ce qui en fait une proto-usine. D'autres formes d'exploitation artisanale, néanmoins, dont les restes sont bien présents dans les forêts du canton, sont généralement représentées par des structures qui attestent d'une exploitation continue mais difficilement sédentaire. Les traces de restauration des structures - par exemple des fours à chaux, comme nous le verrons pourraient être des indices d'une reprise de l'exploitation après une phase où les fours n'ont pas été en activité. Si pour le fer on peut légitimement parler d'activité principale et alternative à d'autres activités comme le pastoralisme, se pose pour d'autres vestiges la question d'en comprendre le rôle et la place occupés dans la vie quotidienne des populations habitant ces territoires.

En appliquant un modèle déjà testé dans d'autres régions européennes ${ }^{10}$ on peut essayer d'expliquer la présence de vestiges d'activités artisanales, par celle que Sylvain Burri définit comme une " économie complémentaire » aux activités du cultum, spécifiquement basée sur l'exploitation de l'incultum $^{11}$. D'après Burri, en Provence (France), les activités traditionnelles liées à l'agriculture et au pastoralisme seraient, à la fin du Moyen Âge et

9. Marion Liвоutet, "Sainte-Croix (VD), l'Auberson », Jahrbuch Archäologie Schweiz, n 95 , 2012, p. 228-229.

10. Voir, par exemple, les recherches de Sylvain Olivier sur la culture du genêt.

11. Voir à ce propos Sylvain BURRI, «Le problème de la mobilité des artisans, pasteurs et usagers de l'incultum en Basse-Provence centrale ( $\mathrm{XII}^{\mathrm{e}}-\mathrm{XVI}^{\mathrm{e}}$ siècle) " in Michel Lauwers, Des sociétés en mouvement. Migrations et mobilité au Moyen Âge, Paris, Publications de la Sorbonne, 2010, p. 131-136 et Sylvain BURRI, «Reflections on the concept of marginal landscape through a study of late medieval incultum in Provence (South-eastern France) ", European Journal of Post Classical Archaeologies, ${ }^{\circ}$ 4, 2014, p. 7-38. 
à l'Époque moderne, concomitantes de toute une série d'activités " artisanales " (exploitation du bois pour la construction de bâtiments et de bateaux, extraction et travail des métaux, production d'objets d'usage quotidien de verre ou de céramique) pratiquées de manière saisonnière, en se basant essentiellement sur le cycle de vie des plantes. Dans son cas spécifique, la comparaison des calendriers des activités agropastorales et celui des activités artisanales montre qu'il existe un lien de complémentarité entre les activités du cultum et celle de l'incultum. Le pastoralisme est, en effet, surtout pratiqué en été, alors que l'incultum, potentiellement exploité tout le long de l'année, l'est surtout lorsque l'activité agricole était moins intense. Il s'agit d'un système circulaire où l'on passe du cultum à l'incultum en fonction des saisons. Ainsi, l'économie de la région analysée par Burri est de type "mixte » et les sociétés agropastorales qui l'habitent se caractérisent par la complexité et la complémentarité des rôles. Les habitants se présentent, dans les sources, comme pasteurs et comme artisans, ce qui témoigne de leur polyvalence. Or, cette interprétation pourrait convenir aux indices présents dans les régions " marginales " du canton de Vaud.

L'importance des activités artisanales pour les zones de marge du canton de Vaud semble se dessiner dans la toponymie des lieux qui composent ces territoires. Les noms que les hommes ont attribués aux territoires peuvent être, dans certains cas, une fenêtre sur le monde physique et cognitif des communautés du passé, un témoignage des façons dont elles s'approprient le paysage dans lequel elles vivent. En plus d'être une indication du lieu topographique, ces noms indiquent les activités humaines de cette portion de territoire ${ }^{12}$. Ainsi, la lecture des cartes historiques dessinant les régions marginales du pays de Vaud montre-t-elle toute une série de toponymes liés aux activités artisanales (Fig. IX et $\mathbf{X}$, cahier couleur). Dans le Jura vaudois nombreux sont les toponymes qui rappellent la production de la chaux (Champ du Raffour ou Combe du Raffort-Raffour et Raffort indiquent, dans le Jura et en Franche-Comté, le four à chaux), l'extraction et le travail du fer (Faverge, Favarge ou Ferreyres) et l'exploitation du bois. Cette dernière est très présente dans le Chablais et dans les Préalpes (Abattes, Apleyau d'où dériverait l'actuel "Les Pléiades " au-dessus de Blonay, ou encore

12. Richard Jones, "Place-names in landscape archaeology ", in Alexandra Chavarria Arnau, Andrew ReYNolds, Detecting and understanding historic landscapes, Mantoue, SAP società archeologica S.R.L, 2015, p. 209-220. 
Chargeoir, Coupée, Retaillons, Taille, Tailla, Taillée, Tailisse). L'exploitation de la pierre est également signalée par les toponymes qui indiquent la présence de carrières pour l'extraction de meules (Molière ou Molleyre), ou de pierre (Perrey, Perrex, Perreire), très présents dans la zone du Chablais et du Jorat. Également, Argilière, Argillat Arzelier, ainsi que Largille, Largilletaz, Larzille ou la forme Erzilière, typique de la Côte et Pied du Jura vaudois, attestent la présence de terrains argileux, fondamentaux pour l'implantation d'une industrie de la terre cuite et de la céramique ${ }^{13}$. Également, les contes et les légendes de la tradition folklorique de ces régions ont souvent des artisans et des bûcherons comme protagonistes, bien qu'ils apparaissent dans une moindre mesure que les pasteurs, les pêcheurs et les chasseurs. Les mineurs et le "beau forgeron Donat " sont les protagonistes de deux légendes combières ${ }^{14}$, respectivement Les mineurs de la Dent de Vaulion et la légende de la Grotte aux Fées ${ }^{15}$. Et le forestier et bûcheron Jacques, avec sa fille Marie, sont les protagonistes de La Fée du Chauderon, légende de la région de Montreux ${ }^{16}$. En proie à des créatures fantastiques telles que les fées, le diable, les gnomes, ces personnages sont l'archétype de l'habitant de ces régions. Malgré la prudence nécessaire lorsqu'on traite de ce genre de sources, les récits imaginaires sont souvent un bon miroir du monde social et identitaire à partir duquel ces contes sont formulés.

Si la toponymie des lieux et la culture folklorique suggèrent, pour les zones marginales vaudoises, la présence d'un substrat économique alternatif, ou du moins complémentaire, aux activités agropastorales, d'autres exemples supportent plus concrètement cette hypothèse.

L'exploitation du calcaire en vue de la production de la chaux, par exemple, a attiré dernièrement l'attention de l'archéologie cantonale suite à la découverte de plusieurs vestiges dans la région du Jura et du Nord-vaudois. Les caractères de cette production, analysés dans un article précèdent ${ }^{17}$, pourraient rentrer dans le cadre théorique dessiné par Burri et ainsi valoriser

13. Maurice Bossard, Jean-Pierre Chavan, Nos lieux-dits. Toponymie romand, Lausanne, Payot, 1986.

14. C'est-à-dire de la Vallée de Joux, au Jura vaudois.

15. Auguste Piguet, La vie quotidienne et les coutumes d'autrefois à la Vallée de Joux : 1944-1949, Les Charbonnières, Éditions Le Pèlerin, 1999.

16. Louis Chardon, Contes et légendes de la forêt. Le temps des fées, Yens, Éditions Cabédita, 1994. 17. Alice Vanetti, Marion Liboutet, "Pour une relecture du statut économique du Canton de Vaud à l'époque moderne : les cas du fer et des fours à chaux du Jura-Nord vaudois ", dans Catherine Leuzinger-Piccand, Urs Niffler (dir.), La Suisse de 1350 à 1850 à travers les sources 
l'hypothèse de la présence d'une économie de type complémentaire également dans le canton de Vaud.

\section{La production de la chaux dans le Jura : une activité complémentaire au pastoralisme?}

La présence de vestiges liés au cycle de production de la chaux dans le territoire du Jura est considérable. Objets de fouilles ponctuelles entre les années 1990 et 2000, essentiellement dans le cadre d'interventions de sauvetage, ces vestiges ont récemment acquis une nouvelle importance pour la recherche suite à l'usage de plus en plus fréquent du Lidar (Light Detection and Ranging) dans l'analyse du potentiel archéologique et historique du territoire vaudois. Appliqué sur le Jura il a permis de déceler une situation encore plus complexe que celle qui avait été livrée par les opérations de prospections et de fouilles. D'après les images, les structures anthropiques interprétables comme des sites de production de la chaux (qui dans certains cas ont pu être vérifiés) sont non seulement plus nombreuses que ce que les opérations archéologiques ordinaires montrent, mais leur position est régie par une volonté bien spécifique (Fig. XI, cahier couleur), l'établissement des sites de production semble en fait répondre plutôt à des exigences d'accès à la matière première et à son transport qu'aux nécessités des communautés locales. Inscrites dans un système d'information géographique (SIG), ces informations, couplées avec d'autres sources telles que les données géologiques, l'inventaire des voies historiques ou les recensements des populations, ont dévoilé les critères des choix des hommes qui ont implanté ici leurs activités : facilité d'obtention des matériaux, mais surtout, facilité de transport du produit (matière première ou produit fini) au lieu de destination, ce qui nous avait conduits à envisager une production à vocation commerciale. Dans la plupart des cas, en fait, ils sont situés loin des habitats. Lorsqu'un habitat peut être signalé à proximité du site de production, sa taille et le nombre d'habitants ne justifient pas la présence de tant de fours. Ils peuvent difficilement renvoyer aux seuls besoins de la communauté (Fig. XII, cahier couleur). Grâce à une comparaison

archéologiques, Actes du Colloque (Bern 25-26 janvier 2018), Basel, Verlag Archäologie Schweiz, 2018, p. 239-252. 
avec les tracés des voies historiques et des sentiers ruraux, il a été possible ensuite de remarquer que la plupart des ensembles de fours se trouvent sur des voies de transport ou dans les environs immédiats. La proximité entre les ressources primaires ainsi que les voies de circulation comme critère principal d'implantation, peut s'expliquer par l'objectif de réduire les coûts de production d'un matériel, tel que la chaux, qui n'était pas hautement rentable sur le marché.

Malgré ces considérations, il paraît difficile d'imaginer pour ces structures une utilisation constante et permanente. Tout d'abord, aucune installation qui atteste d'une présence humaine sédentaire n'a pu être repérée, ni en surface, ni à la suite de la fouille. Dans d'autres cas connus, comme ceux exposés par Rita Vecchiattini dans ses études sur la Ligurie ${ }^{18}$, il a été possible de repérer des habitations - telles que la maison du fornacino permettant le contrôle du four et ainsi son fonctionnement en continu. Dans les cas décrits ci-dessous aucun vestige de construction bâtie ou d'autres indices d'une présence répétée n'ont pu être repérés. Tant le site de Champagne et de Concise, dans le Nord-vaudois, que celui de SainteCroix le Château, dans le Jura, par exemple, se caractérisent par la présence de plusieurs structures par site dédiées à la cuisson de calcaire en vue de la production de chaux vive ${ }^{19}$. Ces fours, de diamètre compris entre 6 et 8 mètres, ont été utilisés de manière prolongée, comme le témoigne la succession de couches de chaux endurée déposées au fond du foyer de la plupart, ainsi que les réparations de la structure réalisée après des cuissons. Ils ont été utilisés, d'après les datations au carbone 14 réalisées sur les échantillons de charbon, entre le $\mathrm{XV}^{\mathrm{e}}$ et le $\mathrm{XVIII}^{\mathrm{e}}$ siècle, et dans certains cas (comme celui de Concise) auraient fonctionné de manière contemporaine. Dans tous les cas, des fronts de carrière ont été repérés à proximité, aux marques de taille évidentes, servant à l'exploitation de la pierre calcaire, nécessaire à la première phase de la chaîne de production de la chaux. Néanmoins, aucune trace indiquant une longue occupation humaine n'a été décelée. Il n'y a trace d'aucune structure domestique ni mobilier. Au

18. Rita Vecchiattini, La Civiltà della calce. Storia, scienza e restauro, Genova, De Ferrari, 2010. 19. Max Kluserner, Four à Chaux de Champagne. Rapport de fouille non publié, Lausanne, Archéologie Cantonale, 1994 ; François EschBACH, Un ensemble de fours à chaux médiévaux et modernes à Concise Vd - Les Favarges. Rapport de fouille non publié. Lausanne, Archéodunum, 1997 ; Anne-Lyse Gentizon Haller, Marc Haller, Léonard Kramer, Tranchées et Sondges d'évaluation Sainte-Croix le Chatteau SC13. Rapport de fouille non publié, Lausanne, Archéologie Cantonale, 2013. 
niveau technique ensuite, la plupart des fours appartiennent au type surface ou semi-enterré, soit des structures relativement basiques et qui ne se prêtent pas facilement à un usage en continu à moins d'y apporter des réparations régulières ${ }^{20}$. Enfin les fronts de carrière présents sur les sites et très abondants dans la région, s'ils témoignent, comme on l'a dit, de l'usage et de l'exploitation sur la longue durée, sont généralement de dimensions modestes et en surface. Ce fait nous induit à envisager une exploitation de ces carrières de manière irrégulière plutôt qu'à y voir l'établissement d'une pratique répétitive.

Si d'un côté il est réducteur de considérer la production de la chaux comme un artisanat voué à la consommation locale ou pour un usage occasionnel (construction ou restauration d'un bâtiment en particulier), d'un autre côté il est difficile de l'identifier comme continue sur l'année. En appliquant le modèle développé par Burri aux territoires de marges vaudois, chaque site de production apparaît comme le résultat d'une exploitation continue sur des courtes périodes mais de manière répétée tout au long de l'année. Ce modèle théorique nous paraît plus approprié. L'application de cette hypothèse est néanmoins compromise par certains détails, notamment en ce qui concerne les différences climatiques et paysagères entre les régions analysées par Burri et les nôtres.

Le climat de la Provence est de type méditerranéen, caractérisé par un faible niveau d'humidité et par des précipitations qui, bien que dans la moyenne nationale, n'atteignent pas des niveaux élevés. Dans ses zones montagneuses, soit celles prises en considération par Burri dans ses recherches, la température moyenne est de 11 à 14 degrés. En hiver, elle est de 3 à 6 degrés, alors qu'en été elle atteint entre 21 et 24 degrés. La végétation se caractérise principalement par la présence du Pin d'Alep et de feuillus comme le chêne. De 500 à 800 mètres, la végétation est dominée par les oliviers. Ces conditions climatiques sont essentiellement favorables à l'établissement d'un cycle de production saisonnier. La rareté de fortes précipitations ainsi que les températures rendent en fait possible, en hiver, lorsque les activités agropastorales sont moins pratiquées, l'implication dans d'autres types d'activités, surtout liées à l'emploi du feu. Le cas du Jura, ainsi que des autres zones " marginales » du canton de Vaud, est

20. Lara Tremblay, "Les fours à chaux du Jura : essai de Typo-chronologie ", Jahrbuch Archäologie Schweiz, n 100, 2017, p. 109-134. 
différent. Les précipitations annuelles sont abondantes, au printemps et en été elles se déclinent par des pluies consistantes qui deviennent, entre la fin de l'automne et l'hiver, de la neige. La température moyenne varie, avec des microclimats qui contredisent parfois les simplifications, entre -5 et 15 degrés. Ces conditions font que l'établissement d'un modèle de production fondé sur un cycle saisonnier à ces régions soit un peu hasardeux. Pourtant, lors des recherches conduites dans la forêt de Chailluz, dans la commune de Besançon, en Bourgogne Franche-Comté (pas très loin du centre de notre analyse) et centrées sur les nombreux vestiges d'activités anthropiques relevés en milieu forestier, les chercheurs avancent des hypothèses qui s'accordent à celles présentées ici. Dans son étude, Catherine Fruchart approfondit la présence, détectée par la prospection mais surtout par l'emploi du Lidar dans la forêt de Chailluz, de plusieurs vestiges archéologiques liés à des activités agropastorales (dont des restes de fermes ainsi que de division des champs) et artisanales comme des plateformes pour charbonnières et des fours à chaux ${ }^{21}$. À travers une analyse spatiale basée sur la comparaison entre la localisation des activités à vocation agropastorales et celles à vocation artisanale, l'auteure avance l'idée que si les trois types de structures comportent " des contraintes d'exploitation et d'occupation du sol différentes ${ }^{22}$ ", leur cohabitation ne peut pas être complètement exclue. En se référant principalement aux charbonnières, Fruchart relève comment l'alternance « en rotation d'une à trois décennies, en particulier au cours de la période médiévale ${ }^{23}$ " d'activités agropastorales et de production de charbon est envisageable dans la mesure où la production du charbon aurait pu contribuer, entre autres, aux pratiques d'essartage en vigueur à l'époque. L'alternance sur des décennies, ou du moins sur des années, d'une pratique d'ailleurs attestée en agriculture, permettrait entre autres d'aller au-delà des contraintes climatiques de ces régions (le département du Doubs et le Jura partagent la même situation) qui nous empêchent d'appliquer entièrement le modèle saisonnier de Burri à notre cas d'étude ${ }^{24}$.

21. Catherine Fruchart, Analyse spatiale et temporelle des paysages de la forêt de Chailluz (Besançon, Doubs) de l'Antiquité à nos jours, Besançon, université de Franche-Comté, 2016, en ligne : https:// hal.archives-ouvertes.fr/tel-01099184v2.

22. Ibid., p. 176 .

23. Ibid., p. 177.

24. En ce qui concerne les fours à chaux repérés dans la forêt de Chailluz, l'auteur relève, par contre, la nécessaire postériorité des fours aux aménagements agropastoraux. 
D'après ces réflexions, la production de la chaux dans le Jura, là où la plupart des sites ont été repérés, pourrait être lue comme une activité complémentaire à d'autres activités principales, comme le pastoralisme ou l'agriculture, et censée apporter des revenus économiques additionnels aux personnes qui s'y adonnaient dans les moments de repos des deux autres activités principales.

La lecture du cas-type de la production de la chaux semble confirmer la présence, dans les zones de marge du canton, d'une économie complémentaire à des activités d'autre type comme celles agropastorales. Certaines sources documentaires, liées toujours à l'exploitation du calcaire et à la production de la chaux, mais également à d'autres matières premières, corroborent cette hypothèse.

\section{Les sources documentaires}

Comme nous l'avons précédemment signalées, les attestations d'un commerce de la chaux au niveau du pays de Vaud sont multiples. Les comptes de restaurations de grands bâtiments, tels que la cathédrale de Lausanne, attestent de l'achat régulier de "chars de chaux " auprès de particuliers ${ }^{26}$. La recherche d'archives a montré que ces particuliers ne sont pas des producteurs de chaux, ou chaufourniers, mais plutôt des intermédiaires qui s'occupent du transport de la chaux jusqu'à la cathédrale et probablement également de l'acquisition du produit même. Ensuite, les lois du pays établies sous la domination bernoise réglementaient la vente de la chaux. Le Coutumier nouveau de la ville et de tout le baillage de Grandson de 1779, par exemple, rappelle par la loi n 206 intitulée « Le Rafournier devra faire taxer sa chaux avant que la vendre étant sur le commun, et ne pourra la distraire ", que "Arrivant qu'un rafournier fasse un rafour à chaux sur le commun, il sera obligé avant que vendre sa chaux de la faire taxer par deux justiciers du lieu [...] et ne pourra l'estimer et vendre au-delà d'icelle taxe ; toutefois, s'il ne pouvoit s'en contenter, il en pourra procurer une révision auprès du Seign. Baillif, et ne pourra la distraire du baillage sans la

25. Alice Vanetti, Marion Liboutet, "Pour une relecture du statut économique du canton de Vaud à l'Époque moderne. Le cas des fours à chaux du Jura/Nord vaudois ", op. cit.

26. ACV Bm 36/1-3, compte général pour les réparations faites à la grande église [cathédrale] de Lausanne. 
permission du Seign. Baillifif ${ }^{27}$. Ces indices reflètent l'existence d'un réseau d'échange de la chaux au niveau régional qui ne peut qu'être supporté par une production réalisée, là où la matière première était présente, dans un objectif " autre " que l'autoconsommation. Si ces documents nous confirment ce que l'analyse territoriale nous avait permis de supposer, d'autres textes nous donnent quelques informations supplémentaires quant à la nature de cette production de chaux.

Un document daté de 1499 dénombrant les usages de la terre de Romainmôtier, soit les droits et les devoirs des hommes libres habitant le territoire qui dépend de l'abbaye clunisienne, énonce que tous les hommes possédant des bêtes de somme et un char ont l'obligation d'assurer les travaux de réparation des édifices de l'église ainsi que des maisons de ses membres et de fournir à ce propos les matériaux nécessaires : des bois de construction, des pierres, de la chaux, du sable, des briques et tout autre matériel ${ }^{28}$.

Un deuxième document, daté de 1679, concernant les dépenses réalisées pour la restauration globale de l'ancienne église abbatiale de Romainmôtier, toujours dans le Jura, atteste l'achat de " 9 fass kalch" (soit 9 tonneaux de chaux) pour le prix de $90 \mathrm{ff} .{ }^{29}$ Le document atteste également le payement à Maître David Poterat (un maître maçon) de $853 \mathrm{ff}$ pour les travaux de restauration, qu'il aurait donc entièrement dirigé. La note semble suggérer que M. Poterat se serait alors également occupé de l'achat du matériel pour la réalisation des restaurations, soit la chaux susmentionnée, mais également du sable (sand) et du gypse (gips).

Or, si ces deux documents nous confirment ultérieurement que la chaux, ainsi que d'autres matériaux de construction comme le sable ou les pierres, étaient effectivement produits dans l'objectif non seulement d'un besoin personnel, mais également d'échange - dans le premier cas le document pose la question d'où ces hommes obtenaient ces matériaux : est-ce qu'ils les produisaient eux-mêmes? Est-ce qu'ils allaient les chercher chez des producteurs spécialisés ? - ils nous informent également sur un autre détail important. Si des figures comme le forgeron, le charpentier, le maçon, sont souvent

27. Coutumier nouveau de la ville et de tout le baillage de Grandson, Yverdon-les-bains, J. J. Hellen, 1780. 28. ACV Fj 137 : "Item, debent dicti homines habentes animalia ad carrucam trahentia charreagium ad edifficia Ecclesiae et mansionum seu membrorum oneri domini cadentium faciendum et reparandum : videlicet, marrini, lapidum, calcis, arenae, tegularum, sinduli et alterius materiei ad edifficia facienda necces saria, quotiens neccesse est et jubetur. »

29. ACV Bp 40/26, comptes du baillage de Romainmôtier. 
explicitement mentionnées dans les documents en tant que professionnels, les producteurs de ces matériaux, dont la catégorie est pourtant reconnue à la même époque dans d'autres pays (nous pensons à la situation des chaufourniers ou des tailleurs de pierre en France, par exemple, réglementés par l'inclusion dans des guildes et des corporations), ne sont presque jamais mentionnés. L'impression est qu'il s'agit de particuliers qui fournissent ces matériaux au besoin, mais qui ne pratiquent pas cette activité assez régulièrement pour être identifiés avec cette profession. D’autres documents plus récents nous confortent dans cette hypothèse, bien que traitant d'autres matériaux que la chaux.

Avec le changement de gouvernement qui suit la Révolution vaudoise et la successive proclamation du canton de Vaud en 1803, nous assistons à la formulation de beaucoup de pétitions adressées par des individus aux communes ou à l'État afin de se voir confirmer des droits d'usage des forêts, alors propriété de ces deux acteurs institutionnels. Ces droits d'usage concernent des activités comprenant l'extraction et la taille de la pierre ainsi que la coupe et le travail du bois. Si dans certains cas cette requête est déposée à la suite de besoins ponctuels (par exemple des réparations dans la maison de l'individu qui fait la demande), dans beaucoup d'autres, l'objectif déclaré est la vente du matériel, brut ou transformé (en charbon, par exemple), dans le canton comme au-delà des frontières, afin de pouvoir subvenir à des nécessités financières. Dans certains cas, ces requêtes coïncident avec des périodes de difficultés économiques ou de manque de travail ${ }^{30}$. Dans le cas du bois, beaucoup de demandes concernent la coupe du bois afin, d'une part, d'installer des charbonnières et, de l'autre, d'alimenter des activités pyrotechniques comme les forges ${ }^{31}$. Or, comme nous l'avons mentionné, ces documents datent du début du XIX ${ }^{\mathrm{e}}$ siècle, lorsqu'une commission des forêts est officiellement créée par l'approbation de la loi forestière (1810) permettant ainsi de réglementer l'usage qu'on faisait de ces zones. Néanmoins la plupart contiennent une requête de confirmation d'un droit qui avait été octroyé, d'après les requérants, par les institutions précédentes. Dans certains cas une référence explicite à la coutume de se servir sur place en vigueur aux époques précédentes, ce qui attesterait alors la durée de cette pratique multiséculaire.

30. ACV K XII b 9, Service des forêts, chasse et pêche - Exploitation de pierre et de terre dans les forêts, dégâts dans les forêts 1803-1836.

31. ACV K XII b 8, Service des forêts, chasse et pêche - Exploitation de bois dans les forêts, 1803-1836. 


\section{Conclusion}

L'incultum vaudois à la fin du Moyen Âge et à l'Époque moderne, pays de montagnes et de forêts, n'est pas une zone marginale et isolée d'après les analyses archéologiques et historiques. Grâce à ses ressources naturelles, il se serait tout au long de l'histoire bien intégré dans l'économie régionale vaudoise. Les bois, ainsi que les minerais et les roches dont ces régions sont riches, ont été longtemps exploités et employés dans tout le canton, ce qui a permis une certaine autonomie des importations externes au moins pour des secteurs comme, par exemple, la construction. Mais c'est surtout au niveau des communautés locales que le rôle de l'incultum est à réévaluer. Considéré par l'historiographie récente comme un lieu principalement exploité par ses habitants en vue du défrichement pour l'obtention de pâturages et des nouveaux terrains agricoles, il aurait au contraire longtemps été une source de revenu et de subsistance pour les communautés locales, tant dans le sens d'une économie de type artisanal professionnel (voir le cas du fer, par exemple), que dans un rapport de complémentarité, avec d'autres activités comme l'agriculture ou le pastoralisme, comme nous le proposons avec la production de la chaux. Cette importance se perçoit d'ailleurs dans les liens qui subsistent encore aujourd'hui, malgré les changements survenus sur le plan technologique, entre habitants et territoire. Les toponymes ainsi que les récits traditionnels en sont un exemple.

L'incultum, avec toutes ses activités multiples, doit être considéré alors comme un vrai substrat économique pour ces régions et, pour utiliser les mots employés par Burri ${ }^{32}$, une composante essentielle de la vie de ces zones rurales à l'époque médiévale et moderne.

\section{L'autrice}

Alice Vanetti est archéologue au Service des immeubles, patrimoine et logistique (SIPaL) de l'État de Vaud (Suisse). Docteure en archéologie de l'université de Neuchâtel (Suisse) et de l'université Bourgogne Franche-Comté (France), ses recherches portent sur l'archéologie du bâti, l'archéologie territoriale et l'histoire des techniques. Contact : alice.vanetti@vd.ch

32. Sylvain BurRI, "Reflections on the concept of marginal landscape through a study of late medieval incultum in Provence (South-eastern France)", op. cit. 